\title{
ESMO: Reshaping the Future of Clinical Trials in Oncology
}

Robin Stannard

Editorial Assistant

Citation: EMJ Oncol. 2021;9[1]:24-26.

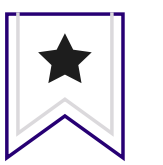

\begin{abstract}
N A MASTERCLASS conducted on Day 5 of the European Society for Medical Oncology (ESMO) Congress 2021, Emiliano Calvo, Director of START Madrid, Centro Integral Oncológico Clara Campal Hospital, Madrid, Spain, and Nathan Cherny, Head of the Cancer Pain and Palliative Medicine Unit, Shaare Zedek Medical Centre, Jerusalem, Israel, presented a joint session on the future landscape of clinical trial practice in oncology, discussing the practical, ethical, and logistical barriers to improving that landscape.
\end{abstract}

\section{PHASE 0 TRIALS}

Opening the session, Calvo presented a compelling and balanced case about the potential that Phase $\mathrm{O}$ (PhO) trials hold for increasing efficiencies in drug development by enhancing the selection of elite drug candidates. Pho studies are carried out very early in the drug development life cycle, even before Phase 1 (Ph1) has occurred. They have limited duration, sample size, and drug dose and have no therapeutic or diagnostic intent. However, these characteristics mean that $\mathrm{PhO}$ trials have reduced regulatory requirements and have the potential to provide key information regarding the pharmacological profile of an investigational new drug (IND) and to streamline the entire drug development process by providing valuable data prior to Ph1.

The number of oncological drugs in development has rapidly increased in recent decades. However, attrition rates remain high with only $10-20 \%$ of drugs making it through the stages of clinical drug development to market. As Calvo explains: "Proportional increases in R\&D [research and development] over the last decades do not necessarily lead to rising numbers of new drugs." This results in increasingly unaffordable drugs and skyrocketing prices that make the entire cancer care system less efficient. The U.S. Food and Drug Administration (FDA) has made efforts to address this issue communicating that one potential method of improving the efficiency of clinical drug development would be investment in preclinical studies, which enhance predictability of IND clinical trial success. PhO trials are a method of enhancing this predictability.

PhO trials can be categorised depending on what they are investigating. Microdose PhO trials investigate pharmacokinetics (PK) by collecting early information on how the body processes the drug. These studies typically use $1 \%$ of a pharmacologically active dose to profile the drug targets and pharmacological effect without eliciting any adverse consequences. The second 
type of Pho trial focuses on the study of pharmacologically relevant doses, known as pharmacological endpoint studies. These studies involve treating with varied dose levels over a very short window, often 7 days. The final category refers to the study of the mechanism of action or pharmacodynamic PhO trials. This category of PhO is used as a proof of concept for drug mechanisms, measuring factors such as degree of receptor saturation, inhibitions of active enzymes, or other 'biomarkers' of drug activity. The doses are incredibly low as only tumour-related pharmacodynamic effects are measured; these studies should produce no toxicity and no therapeutic effects. Calvo emphasised that this third, mechanism of action, PhO trial may prove to be the most relevant in the era of precision medicine.

\section{"If you do all these things, you won't be making the same mistakes that researchers have been making over the last 15 years"}

Whilst Calvo placed emphasis on the potential PhO trials offer to increase predictability, streamlining, and eventual efficiency and frugality in the clinical drug development process, he also offered a balanced argument of the issues that incorporating $\mathrm{PhO}$ trials into general practice may present. Technologically, PhO trials are challenging, as detecting drug impacts at such low doses within patients is very difficult and there are few sites with the necessary equipment for these processes. Furthermore, even though some PhO trials have reduced regulatory requirements compared to Ph1, this may not always be the case depending on the nature of the PhO. However, possibly the most important challenge that $\mathrm{PhO}$ trials present is not technological but ethical. As Calvo explained, it could be argued that improved development efficiencies resulting in reduced cost and time provide large-scale practical benefits to cancer care. However, PhO trials are so short-term and low-dose that there is no therapeutic benefit to the individual participant, meaning that these studies are more about the drug than the patient. Calvo emphasised that as a physician with a duty of care, if there is another Ph1 trial available to the patient, this must be recommended as it is in the patient's best interest. Calvo aptly summarised the dilemma of this scenario by stating: "patients come to oncologists to fight their cancer but [the] oncologist is offering no fight at all".

Calvo ended his presentation with an important reflection of how to consider the future of $\mathrm{PhO}$ trials, which is to return to the initial question: 'Do they improve the success rate of human trials entering drug development?' He summarises that currently it is difficult to conclusively decide, but that failure of a drug early must always be better than failure later. He emphasised that improving the ethical feasibility of PhO trials would be necessary by considering suggestions such as amending Ph1 studies to allow participants from PhO trials or selecting clinically stable patients during mandatory systemic therapy vacations.

\section{SINGLE-ARM STUDIES}

Cherny, a serving member of the ESMO Designated Centre Working Group and the ESMO Magnitude of Clinical Benefit Working Group, undertook a more retrospective analysis. By taking a critical approach to practices currently harming clinical trials in oncology, he provided a convincing argument on why these practices should be left in the past and not incorporated into the future. Cherny began his presentation with a discussion on research integrity and the minimisation of bias, conceptualising the harm bias presents in three ways: direct harm through misleading outcomes; societal harm through inappropriate resource allocation; and reputational harm through loss of credibility. Establishing these directives of research integrity led him seamlessly and pertinently into his critical analysis of single-arm studies (SAS).

In a SAS all enrolled participants are treated with the experimental therapy, in contrast with a randomised controlled trial (RCT) where randomly selected groups of patients are treated with different therapies to compare medical outcomes. The FDA defines SAS as acceptable for drug approval "in settings where there is no available therapy and where major tumour regressions can be assumed to be attributed to the test drug." However, in recent years SAS have been accepted as proof of efficacy in several situations such as when standard treatments 


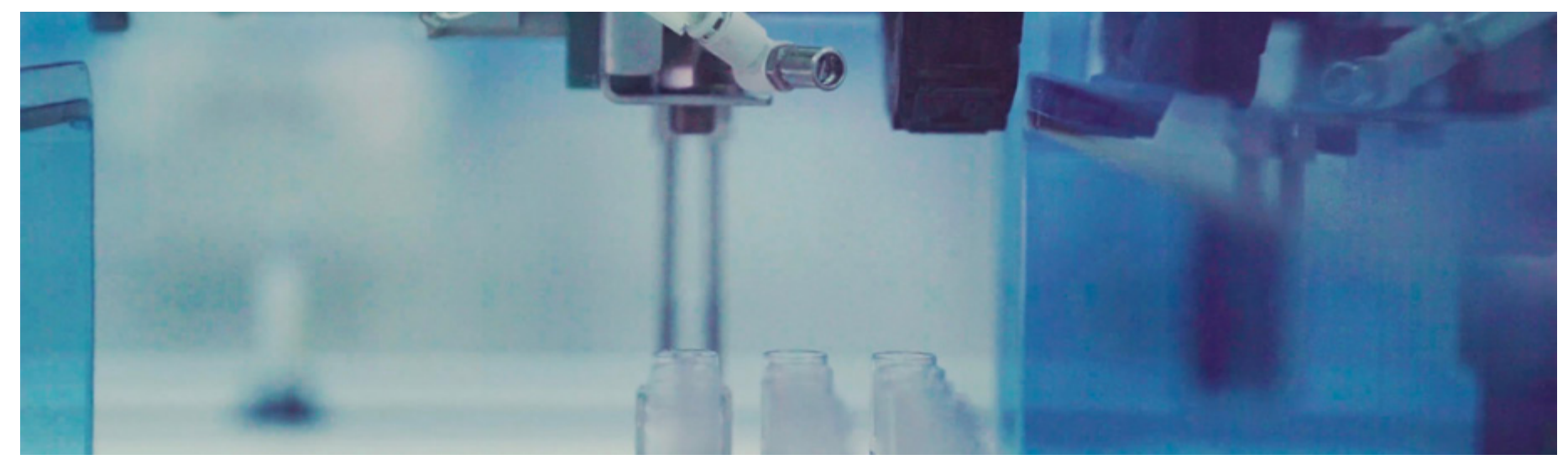

do not exist or are clearly inferior, when the disease is rare, or when patient accrual for RCTs is not perceived to be feasible.

Cherny explained that SAS are much less reliable as evidence for patient benefits and are frequently being employed despite the existence of a reasonable alternative therapy or when there is no evidence for the infeasibility of an RCT. Using evidence from a meta-analysis of clinical trial findings, Cherny demonstrated how overall response rates (ORR) in solid tumour SAS are higher than the ORR when the same medicine for the same indication is tested in a RCT. The meta-analysis evaluated the average gap in ORR between SAS and RCT, finding that SAS were on average $12.9 \%$ higher in 2005 and $8 \%$ higher in 2020.2 Cherny described the overly optimistic findings of SAS as "an issue of generalisability and reproducibility."

\section{"Possibly the most important challenge that Pho trials present is not technological but ethical"}

An additional concern with SAS is that they are being used inappropriately. Rittberg et al. ${ }^{3}$ conducted an evaluation of 31 drug approvals based on the outcomes of SAS, finding that there was an alternative drug that could have been used as a control arm available in 28 out of those 31 cases. In five cases the drugs were approved despite demonstrating inferior efficacy compared to the standard of care and in $>85 \%$ the authors summarised that it would have been feasible to complete an RCT within a reasonable time frame. Cherny agreed with the authors' conclusion that this equated to bad science, with SAS generating accelerated approvals that resulted in subsequent rescindments or drugs that lingered in the market with no definitive proof of benefit.

In his concluding remarks, Cherny revisited his initial position about the value and importance of research integrity: "wasteful research is not ethical; it is not ethical to recruit patients to participate in studies that are not going to generate generalisable knowledge."

\section{CONCLUDING COMMENTS}

The arguments presented by both experts provided an interesting balance between logistical concerns including increasing efficiencies, saving money, and the availability of therapies, versus the ethics of providing optimum care, practising research integrity, and prioritising the patient over the disease. The retrospective analysis alongside a look to the future demonstrates the importance of balancing these two factors: striving to learn from mistakes, and consider improvements and innovations for the future. In the Questions and Answers at the end of the session, Cherny summarised this emerging theme: "If you do all these things, you won't be making the same mistakes that researchers have been making over the last 15 years. And the quality of the research and integrity of the research you produce is going to be of a different standard."

\section{References}

1. FDA. Clinical trial endpoints for the approval of cancer drugs and biologics. 2018. Available at: https:www.fda.gov/ media/71195/download. Accessed 9 October 2021

2. Zia Ml et al. Comparison of outcomes of phase II studies and subsequent randomised control studies using identical chemotherapeutic regimens. J Clin Oncol. 2005;23(28):698291.

3. Rittberg $\mathrm{R}$ et al. Feasibility of randomized controlled trials for cancer drugs approved by the food and drug administration based on single-arm studies. JNCl Cancer Spectr. 2021;5(4):pkab061 